\title{
Article \\ Machine Learning Based Predictions of Fatigue Crack Growth Rate of Additively Manufactured Ti6A14V
}

\author{
Nithin Konda, Raviraj Verma and Rengaswamy Jayaganthan *
}

check for updates

Citation: Konda, N.; Verma, R.; Jayaganthan, R. Machine Learning Based Predictions of Fatigue Crack Growth Rate of Additively Manufactured Ti6Al4V. Metals 2022, 12, 50. https://doi.org/10.3390/ met12010050

Academic Editor: Yves Nadot

Received: 22 October 2021

Accepted: 1 December 2021

Published: 27 December 2021

Publisher's Note: MDPI stays neutral with regard to jurisdictional claims in published maps and institutional affiliations.

Copyright: (C) 2021 by the authors. Licensee MDPI, Basel, Switzerland. This article is an open access article distributed under the terms and conditions of the Creative Commons Attribution (CC BY) license (https:// creativecommons.org/licenses/by/ $4.0 /)$.
Department of Engineering Design, Additive Manufacturing Group \& Centre of Excellence for Materials and Manufacturing for Futuristic Mobility, Indian Institute of Technology Madras, Chennai 600036, India; ed19s015@smail.iitm.ac.in (N.K.); rverma1@mt.iitr.ac.in (R.V.)

* Correspondence: edjay@iitm.ac.in; Tel.: +91-44-2257-4735

\begin{abstract}
The present work focusses on machine learning assisted predictions of the fatigue crack growth rate (FCGR) of Ti6Al4V (Ti64) processed through laser powder bed fusion (L-PBF) and post processing. Various machine learning techniques have provided a flexible approach for explaining the complex mathematical interrelationship among processing-structure-property of the materials. In the present work, four machine learning (ML) algorithms, such as K- Nearest Neighbor (KNN), Decision Trees (DT), Random Forests (RF), and Extreme Gradient Boosting (XGB) algorithms are implemented to analyze the Fatigue Crack growth rate (FCGR) of Ti64 alloy. After tuning the hyper parameters for these algorithms, the trained models were found to estimate the unseen data as equally well as the trained data. The four tested ML models are compared with each other over the training as well as testing phase, based on their mean squared error and R2 scores. Extreme Gradient Boosting has performed better for the FCGR predictions providing least mean squared errors and higher R2 scores compared to other models.
\end{abstract}

Keywords: titanium alloy; additive manufacturing; machine learning; fatigue crack growth rate

\section{Introduction}

Ti6Al4V is known for its high specific strength, fracture toughness, high temperature mechanical properties, and excellent corrosion resistance properties. Consequently, it is widely adopted in aerospace and biomedical applications which predominantly are geometrically complex shapes. This material is responsible for higher tool wear and worse machined surface integrity [1-3]. Many cutting tool materials are in fact chemically reactive to the titanium alloys, which makes it difficult to machine using traditional methods. To address these problems, apart from traditional manufacturing methods, Laser Powder Bed Fusion (L-PBF) has become a popular metal additive manufacturing method for producing geometrically complex shapes [4-6]. L-PBF is an additive manufacturing process in which successive layers of powder are selectively melted by the interaction of a highenergy-density laser beam. The successive layers consolidate to form the required solid shape. After the printing process, to improve the surface properties and enhance the microstructural homogeneity, surface and heat treatments such as hot isostatic pressing, shot peening, and laser shock peening are performed over the as built samples [7-10]. As this manufacturing technique allows the fabrication of more intricate geometries, it is used in aerospace and biomedical applications. Especially in aerospace applications, some of the components such as spars, ribs, and longerons are made of Ti alloys which experience high vibrations. Due to these rigorous operating conditions, vibrations lead to fatigue-induced failures, which reduces the useful life of the component [11-15]. On account of few inherent defects in additively manufacturing methods, such as poor bonding defects, porosities, and edge crack initiations, makes the components vulnerable to cyclic loading. E. Pessard et al. [16] have studied the effect of pores and tested volume size on fatigue performance of additively manufactured Ti6Al4V alloy. The different number of 
hemispherical surface holes are introduced to locally raise the stress and change the size of highly stressed volume. In their study on the relationship between thermal expansion and residual stresses, M. Yakout et al. [17] concluded that the volumetric laser energy density could be used as a design tool for identifying part flaws expected during the LPBF process. Processing parameters hence play a huge role in determining the microstructure as discussed in Section 5, as it eventually affects the fatigue performance of the material. Y.W. Luo et al. [18] have adopted a machine learning approach to predict the fatigue life of IN718 alloy influenced by pores, which revealed that increase in the size and the number of pores and decrease in the distance from the center of the pore to the surface of the specimen may promote fatigue crack initiation and therefore could decrease the fatigue life. Hence, it is important to understand how the fatigue crack grows, which in succession would decide material performance so as to avoid catastrophic disasters occurring due to dynamic loading conditions $[4,19]$.

Stress Intensity Factor (SIF) is the most important parameter that is used in fracture mechanics for estimating the fatigue crack growth rate. The crack growth rate $(\mathrm{da} / \mathrm{dN})$ is dependent on the stress intensity factor range $(\Delta \mathrm{K})$, which in turn depends on the crack size (a). The governing equation used to characterize the FCGR problem is the Paris law $[20,21]$. The plot from the crack growth rate versus $\Delta \mathrm{K}$ contains predominantly three regions: region 1 is the near-threshold or crack initiation region in which the curve becomes steep and approaches asymptote $\Delta \mathrm{K}$ th, which is the lower limiting $\Delta \mathrm{K}$; region 2 corresponds to the stable macroscopic crack growth; and region 3 corresponds to unstable crack growth just before final failure and is controlled primarily by the fracture toughness of the material. Other than Paris law, two more relationships are used to estimate the FCGR, which are the NASGRO equation and Walker equation. These two equations account for the stress ratio, which is not accounted for in Paris equation. In this study, the data plotted using the Paris equation is analyzed and used for predictions using ML models.

During crack nucleation, fine microstructures are favorable because of the lower quantity of long slip bands. This causes lesser irreversible slipping to occur, which leads to fewer nucleation sites. Also, the morphology of defects will affect the crack nucleation stage. The stress risers in the vicinity of the crack affect the slip activation rate indirectly. The LPBFed materials propagating cracks are perpendicular to the built layers and the exact direction as the orientation of the elongated grains. It is evident from the SLM fabricated Ti6Al4V samples that columnar grain structure is visible as a result of successive layer deposition [22]. The as-built (AB) sample exhibits martensitic morphology consisting of $\alpha^{1}$ plates. When stress is relieved to $650^{\circ} \mathrm{C}$, the $\mathrm{AB}$ martensitic structure partially decomposes towards acicular $\alpha$. When heat-treated to $890^{\circ} \mathrm{C}$, the reformed $\beta$ phase transforms upon cooling to Widmanstätten $\alpha / \beta$ structure. L. Thijs et al. [23] investigated the microstructural evaluation during laser powder bed fusion of Ti6Al4V and reported that the direction of the elongated grains depends on the local heat transfer condition, which is determined by the scanning strategy and is the independent variable for this current study. These microstructural features ought to be optimized for fatigue design and the manufacture of components using additive manufacturing technology. The microstructure and property correlation are discussed in Section 5.

The fatigue experiments are highly expensive and time-consuming, especially in the case of the Ti64 alloy, which is very costly. In recent times, because of the improved computing power and data storage capacities, data handling and analysis have become exponentially faster and cheaper. Therefore, advanced data interpretation methods have been used to solve the complex nonlinear damage mechanics problems. Machine learning (ML) algorithms are capable of estimating the patterns available in the data, classifying the data, and clustering, each finding its application in the mechanical and material science fields. The literature shows that the data-driven modeling has been used for estimating the mechanical properties and predictions in the area of fatigue and fracture mechanics [24-26]. As the availability of data becoming easier, many researchers are exploring the adoption of Machine learning and Deep learning models to accelerate the predictions of material 
properties. Using a backpropagation neural network (BPNN) and extreme learning machine (ELM), Raja et al. [24] have estimated the FCGR of Al 2014 alloy and concluded that ELM performed better on the dataset. Nguyen et al. [25] predicted the fatigue crack propagation, adopting deep learning models such as the multi-layer neural network and long-short term memory method, and found out that the multilayer neural network is less effective than the long-short term memory method. Wang et al. [26] compared three machine learning algorithms, which were the ELM, radial basis function network (RBFN), and genetic algorithms optimized backpropagation network (GABP) to predict FCGR of Al2024T351 alloy. Z. Zhan et al. [27] have used ANN and RF models for fatigue life predictions of Additively Manufactured AlSi10Mg, Ti6Al4V, and SS316L. They [28] have analyzed the effects of processing parameters on Additively Manufactured SS316L using Multi Layered Perceptron algorithm and compared it with SVM and RF models. S. Moon et al. [29] utilized the dropout neural networks to understand the impact of surface and the pore characteristics on the fatigue life of laser powder bed fusion fabricated Ti6Al4V alloy. However, the chosen variables which influence the crack growth rate are different in each case.

In this work, the FCGR of Ti64 fabricated through LPBF is predicted using K- Nearest Neighbors (KNN), Decision Trees (DT), Random Forest (RF), and Extreme Gradient Boosting algorithms (XGB). The prediction using the KNN algorithm, in general, is based on the unknown's nearest neighbors. The nearest neighbors can be tuned based on the requirement. The decision trees are the tree-based algorithm where the data is split based on the lowest mean squared error, whereas the other two algorithms are used as ensemble techniques that construct multiple decision trees which collectively make the predictions. These tree-based algorithms predominantly are used to identify the nonlinearity present in the data set. The experimental FCGR data of Ti-64 alloy reported by V. Cain et al. [22] were taken for the analysis using ML models. The independent variables include the post-processing technique, the built orientation during fabrication, and the stress intensity factor. These three parameters significantly influence the FCGR and are independent of each other [3,11,30-33]. Given these three independent variables, the ML models are used to estimate the FCGR of Ti-64 alloy. After modeling the data, the performance of the models was evaluated and the best performing algorithm was employed to carry out the feature importance analysis. Feature importance analysis provides the rankings of the independent variables based on their importance. This analysis showed that SIF is the most influencing parameter followed by post processing technique and built orientation for the FCGR behavior of Ti64 alloy fabricated using LPBF.

\section{Data Description}

In order to build the ML models, relevant experimental Fatigue data of Ti-64 alloy is collected from the literature. The experimental studies on Ti-64 alloy contain standard compact tension (CT) specimens were manufactured from grade 5 Ti6Al4V spherical powder for the determination of FCGR properties. The powder particle sizes ranged from $15 \mu \mathrm{m}$ to $45 \mu \mathrm{m}$. FCGR experiments were conducted as per the ASTM E647 standard. Prior to commencing the FCGR evaluation, a $1 \mathrm{~mm}$ long pre-crack was developed and machined using EDM. The cyclic loading at $5 \mathrm{~Hz}$ and Stress Ratio 0.1 during testing conditions were reported by V. Cain et al. [22], by varying three different built orientations, such as XY, $\mathrm{XZ}$, and $\mathrm{ZX}$, and with three different post processing techniques, such as as-built, stress relieved, and heat treated. The stress relieved specimens were soaked at $650{ }^{\circ} \mathrm{C}$ for few hours, and heat treated specimens were subjected to annealing treatment at $890^{\circ} \mathrm{C}$ for $2 \mathrm{~h}$. The process parameters were optimized and the post-processing parameters mentioned above were collected from the literature [22]. The data from the Paris law graphs for each different processing and post-processing condition are collected and used for the predictive analysis of the FCGR of Ti64 alloy. The design of experiments is shown in Table 1. 
Table 1. Experiments Design [22].

\begin{tabular}{cc}
\hline Post Processing Technique & Built Orientation \\
\hline As Built & $\mathrm{XY}$ \\
Stress Relieved & $\mathrm{XZ}$ \\
Heat Treated & $\mathrm{ZX}$ \\
As Built & $\mathrm{XY}$ \\
Stress Relieved & $\mathrm{XZ}$ \\
Heat Treated & $\mathrm{ZX}$ \\
As Built & $\mathrm{XY}$ \\
Stress Relieved & $\mathrm{XZ}$ \\
Heat Treated & $\mathrm{ZX}$ \\
\hline
\end{tabular}

For these 9 different specimen conditions, the tests are conducted and Paris curves were plotted for each of the experiments. The data was collected for each of the 9 different specimen conditions and data points were taken from the Paris law curves, which were plotted. The data points which were collected are plotted for visualization during the analysis and shown in the Figure 1.
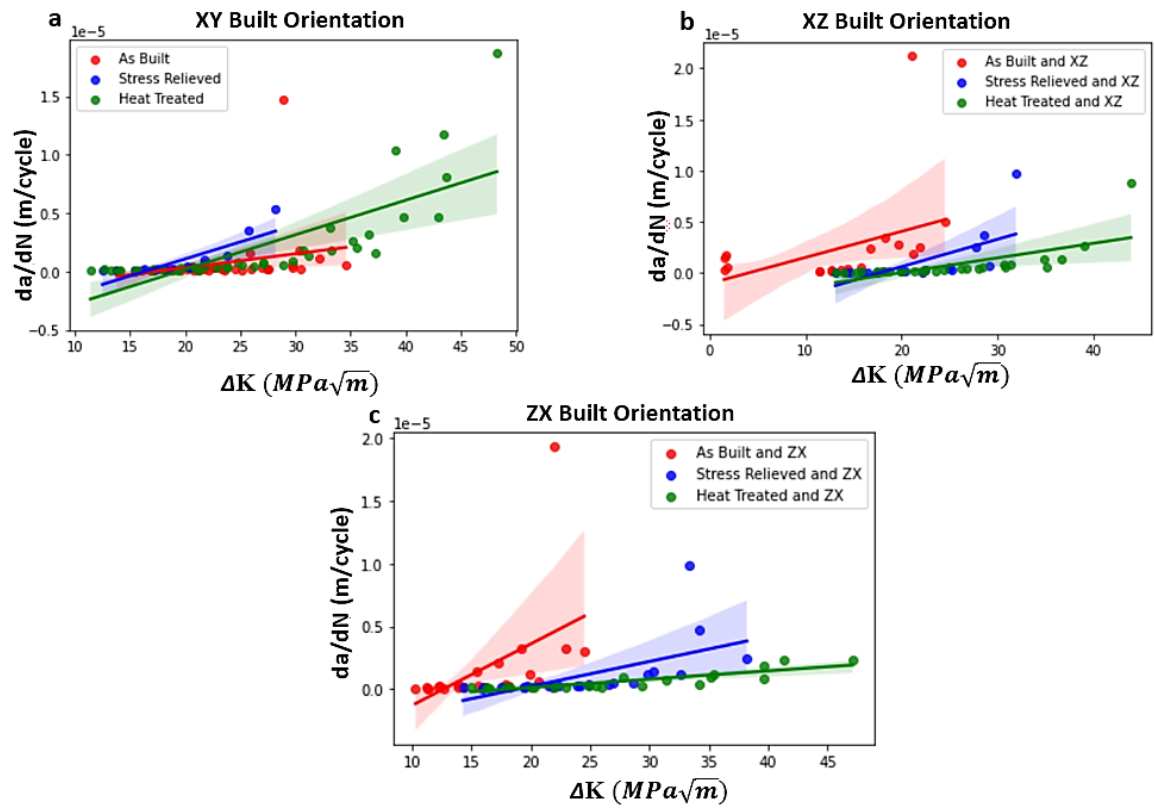

Figure 1. Experimental FCGR data in Ti-64 alloy (a) XY Specimen Orientation; (b) XZ Specimen Orientation and (c) ZX Specimen Orientation.

From the above graphs, it is observed that the effect of build direction is noticeable when compared to the stress relieved and heat treated specimen conditions. The FCGR is drastically increases when the build orientation is changed from $X Y$ to $Z X$ or $X Z$, whereas, for the heat-treated specimen, the crack growth rate decreases when the built orientation is changed from $\mathrm{XY}$ to $\mathrm{XZ}$ or $\mathrm{ZX}$. On the other hand, when we analyze the influence of post processing, the as-built specimen has performed better than the stress relieved and heat treated specimens in XY built orientation, showing an increase in FCGR in the XZ and ZX built orientations.

\subsection{Model Building}

The individual data points in Figure 1 are considered as one data point. For each individual data point in the graph, a corresponding output is generated. In this manner, the dataset is formed for each of the nine experiments, which are shown in Table 1. This data set is utilized for model building and predictions of FCGR for the Ti64 alloy. 


\subsection{Feature Selection}

For building a predictive model, the feature selection plays a major role which eventually contributes to the target value prediction. All the variables cannot be included in the data; the variables that are mostly influencing the target variable, which is FCGR for this study. Based on the domain knowledge and looking at the graphs in Figure 1, we can presume that the post-processing technique and build orientation could be independent variables for the analysis. Additionally, from the Paris law graphs, $\Delta \mathrm{K}$ would be another independent parameter. The sample data points after preparation of the data set are shown in Table 2.

Table 2. Sample of collected data for Ti-64 alloy.

\begin{tabular}{cccc}
\hline Post Processing & Built Orientation & $\Delta \mathbf{K}(\mathbf{M P a} \sqrt{\mathbf{m}})$ & Crack Growth Rate $(\mathbf{m} /$ Cycle $)$ \\
\hline As Built & $\mathrm{XY}$ & 20.7 & $3.87 \times 10^{-7}$ \\
\hline Heat Treated & $\mathrm{XZ}$ & 22.5 & $2.83 \times 10^{-7}$ \\
\hline Stress Relieved & $\mathrm{ZX}$ & 14.3 & $1.05 \times 10^{-7}$ \\
\hline
\end{tabular}

\subsection{Model Development}

A series of models were built for the nine different specimen conditions, which are shown in the Table 1, for the FCGR predictions of Ti6Al4V alloy using four different algorithms. The train and test data are split into $85 \%$ and $15 \%$, respectively, where the train data is used for building model and hyper parameter optimization and the test data are used to check performance of the model on the unseen data. The data has been split into train and test datasets as there are no experimental results to validate the model that was built other than the test dataset, whereas, the model is validated by comparing the performance of the model on the train and test datasets to verify whether the model is overfitting or underfitting in the present work. The scikit-learn library was utilized for adopting algorithms, which are optimized algorithms [34]. A brief mathematical understanding of each algorithm is provided in the following section.

\subsubsection{K-Nearest Neighbour Algorithm}

Among all the ML Algorithms, KNN is the simplest algorithm. This algorithm can be used for both classification and regression problems. This algorithm uses feature similarity to predict the values of unseen data points. The new data point is assigned a prediction on how closely it resembles the data points in the training dataset. For this dataset, as this is a regression problem, the average value of the $\mathrm{k}$ nearest neighbors in the training data is taken as a prediction for the unseen data. There are few methods to calculate nearest neighbors of the smallest distance, including the Euclidean distance, Manhattan distance, and Hamming distance. For this study, we have used the Euclidean distance for calculating the distances between the data points. The Euclidian distance ' $d$ ' is calculated by the formula shown below in the Equation (1):

$$
d=\sqrt{\sum_{i=1}^{k}\left(x_{i}-y_{i}\right)^{2}}
$$

where $x_{i}$ is the new point and $y_{i}$ is the existing point. $\mathrm{K}$ value in this algorithm is the hyperparameter which has to be tuned to get the better result. For this problem, the value of $k=3$ has got least mean squared error when compared to other $k$ values, which tells us that taking 3 nearest neighbors and averaging the value gives us the better results.

\subsubsection{Decision Trees}

A Decision Tree is a supervised machine learning algorithm used for both classification and regression problems. A decision tree segments the features by applying a set of decision 
rules. After segmenting the feature space, a constant piecewise approximation, such as the mean, is used for predictions. Starting with the parent node containing the whole training data, the decision tree is built by recursive partitioning. Recursively, the nodes are split based on the minimization of mean squared error on the child nodes. The minimization function for splitting the nodes is as follows.

$$
\begin{gathered}
\frac{1}{N_{A}} \cdot \Sigma_{A}\left(Y-\hat{Y}_{A}\right)^{2}+\frac{1}{N_{B}} \cdot \Sigma_{B}\left(Y-\hat{Y}_{B}\right)^{2} \\
\hat{Y}_{A}=\frac{1}{N_{A}} \cdot \Sigma_{A} Y_{i} \text { and } \hat{Y}_{B}=\frac{1}{N_{B}} \cdot \Sigma_{B} Y_{i}
\end{gathered}
$$

where, $Y$ is the predicted value in corresponding child node, $\hat{Y}_{A}$ is the average of the predictions in the child node $\mathrm{A}$ and $\hat{Y}_{B}$ is the average of predictions in the child Node B. Decision trees are interpretable and versatile and able to identify complex relationships. In spite of being able to identify the nonlinear patterns, decision trees often tend to overfit. A small variation in the dataset which leads to a significant change in the tree structure could alter the overall predictions. To avoid overfitting, the tree structure should be controlled by providing the hyperparameters such as constraining the depth of the tree, minimum samples in the leaf nodes etc. In the present work, Decision trees with a maximum depth of 6 provides the best results on train and test datasets.

\subsubsection{Random Forest}

Random Forest is the tree-based ensemble method which is used for both classification and regression problems. To overcome the limitations in Decision Trees, the random forest algorithm is trained through bagging (bootstrap aggregation), which is an ensemble approach that improves the accuracy of the CART algorithm. In bagging, the decision trees are built in parallel based on the feature and data sampling. Multiple decision trees are built on those respective samples; hence, for achieving an overall better model, this algorithm results in a greater diversity of trees which trades on a higher bias for lower variance. In regression problems, this algorithm establishes the outcome based on the predictions of the decision trees which are built in parallel. The prediction of the unseen data is the average of the outputs from various decision trees. Increasing the number of trees increases the precision of the outcome. It is more accurate than decision trees, gives reasonable predictions without hyper-parameter tuning, and also solves the issue of overfitting in decision trees.

\subsubsection{Extreme Gradient Boosting Algorithm}

Extreme Gradient Boosting (XG Boost) is an optimized Gradient Boosting algorithm which is highly efficient and flexible to most datasets. This algorithm uses a regularization term which makes this algorithm robust [35]. The objective function which ought to be minimized is the sum of loss function and regularization term, shown in the below equation.

$$
L=\sum_{i} l\left(y_{a}, y_{p}\right)+\Sigma_{k} \beta\left(f_{k}\right)
$$

where $L=$ loss function representing the difference between the actual target value and the predicted target value and $\beta\left(f_{k}\right)$ is the regularization term.

$$
\beta\left(f_{k}\right)=\gamma T+0.5 \cdot \lambda \cdot \omega^{2}
$$

where $T$ represents, number of trees, $\omega$ represents, the leaf weight, $\gamma$ represents, the pruning index, and $\lambda$ is the scaling factor of the weights, which are the hyper parameters. Since the residuals of the preceding regression tree are trained to the succeeding regression 
tree, the objective function at $\mathrm{t}$-th instance gets an additional term $f_{t}\left(x_{i}\right)$ as shown in the below equation.

$$
L=\sum_{i} l\left(\left(y_{a}, y_{p}^{t-1}\right)+f_{t}\left(x_{i}\right)\right)+\beta\left(f_{t}\right)
$$

As this is an objective function which is the function of functions, traditional optimization methods in Euclidian space cannot be used for solving this problem. Hence, we use Taylor's approximation to transform that objective function to a function in Euclidean domain, which helps us to use traditional optimization techniques. First and second terms of the Taylor's approximation are taken and other terms are eliminated. The equation is given below.

$$
L=\sum_{i} l\left(\left(y_{a}, y_{p}^{t-1}\right)+g_{i} f_{t}\left(x_{i}\right)+(0 \cdot 5) h_{i} f_{t}^{2}(x)\right)+\beta\left(f_{t}\right)
$$

where $g_{i}$ is the first derivative of the loss function and $\mathrm{h}_{\mathrm{i}}$ is the second derivative of the loss function. Finally, after removing the constants, the loss function becomes,

$$
L=\sum_{i}\left(g_{i} f_{t}\left(x_{i}\right)+(0 \cdot 5) h_{i} f_{t}^{2}(x)\right)+\beta\left(f_{t}\right)
$$

The above loss function needs to be minimized and next decision tree is built based on the errors predicted in the previous trees. Hence, eventually patterns are identified when the residuals approach zero.

\subsection{Hyper Parameter Optimization}

Hyper parameter tuning is necessary to improve the performance of the model. Especially in decision tree-based models when the hyperparameters are kept default, the model tries to build deeper trees which eventually gives better results on the train data but performs poorly on test data. Hence, to restrict model to overfit and to generalize the model, hyper-parameters are tuned. In order to obtain the best set of hyper-parameters that predicts the FCGR behaviour closest to the experimental values, Bayesian optimization is performed over the hyper-parameter space to minimize, the root mean squared error. Let $\mathrm{H}$ be the overall hyperparameter space, ' $\mathrm{h}$ ' be the vector in $\mathrm{H}$ and ' $\mathrm{O}$ ' be the objective function that has to be minimized. In the Bayesian approach, $\mathrm{P}(\mathrm{O} \mid \mathrm{h})$ is used for hyper-parameter sampling and updated iteratively to obtain optimum hyper-parameters. The least root mean squared error is obtained in XGB, hence the hyper parameters were optimized for that algorithm using Optuna package. The optimized hyper parameters for XG Boost algorithm obtained after Bayesian Optimization are shown in Table 3.

Table 3. Optimized Hyperparameters for XGB.

\begin{tabular}{cc}
\hline Hyper Parameters & Values \\
\hline Booster & Dart \\
Learning Rate & 0.084 \\
Maximum depth & 3 \\
Estimators & 96 \\
Alpha & 0.188 \\
Lambda & 0.0088 \\
\hline
\end{tabular}

Figure 2 represents the optimization process graphically, adopted for the FCGR modelling. As can be seen, the objective value approaches the best value more closely as the number of trials keeps increasing. 


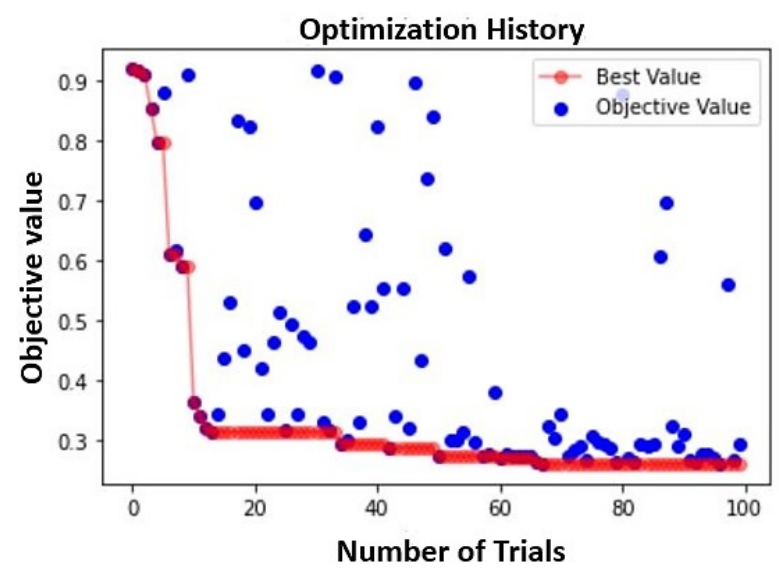

Figure 2. Optimization History Plot.

\section{Methods}

\subsection{Model Validation}

After developing the models, the predicted results are compared with the actual data. To analyze this, the experimental and predicted curves are plotted together to visualize the closeness of the data points. When the curves of experimental results and predicted results approach closer to each other, the predictive ability becomes stronger. The closeness of the 9 different specimen conditions is plotted using the XGB model, which gives the best results for this dataset. The actual and predicted results are plotted below.

The performance of the developed models was analyzed using few metrics. As this was a regression problem, the metrics were the mean squared error, mean absolute error, and $R_{2}$ Scores. The model was trained on the train data and the performance was checked on the test data. In order to check if the model was over-fitting or under-fitting, the performance is checked both on train and test data. The performance metrics formulae are shown in the equations provided below.

$$
\begin{gathered}
\text { mse }=\frac{1}{N}\left[\sum\left(y_{a}-y_{p}\right)\right]^{2} \\
\text { mae }=\frac{1}{N} \Sigma\left|y_{a}-y_{p}\right| \\
R_{2}=1-\frac{\sum\left(y_{a}-y_{p}\right)^{2}}{\sum\left(y_{a}-y_{m}\right)^{2}}
\end{gathered}
$$

where, $y_{a}$ is the actual value, $y_{p}$ is the predicted value and $y_{m}$ is mean of the actual values.

The Paris Curves in general have 3 regimes which are crack initiation region, stable crack growth and unstable crack growth. In these three regimes, the crack initiation and unstable crack growth are non-linear when observed from the curves, whereas the stable crack growth region which is region 2 is linear. When the graph plotted is converted to mathematical terms which is:

$$
d a / d N=C^{*} \Delta K^{m}
$$

which is exponential curve which accounts for linearity in all the three regions when it is converted to logarithmic scale, where as it is evident from the Figures $3-5$ that the nonlinearity is accounted for the 1st and 3rd regimes only using the developed ML model. Hence, the actual graphs can be replicated given the features that account for the FCGR. The models that are developed are compared using some of the performance metrics. When the $R_{2}$ score for the test data and train data are almost similar, then the model is said to be performing similar to the train data on the unseen data. The performance measures are plotted for all the 4 algorithms and compared as shown in the figures below. 

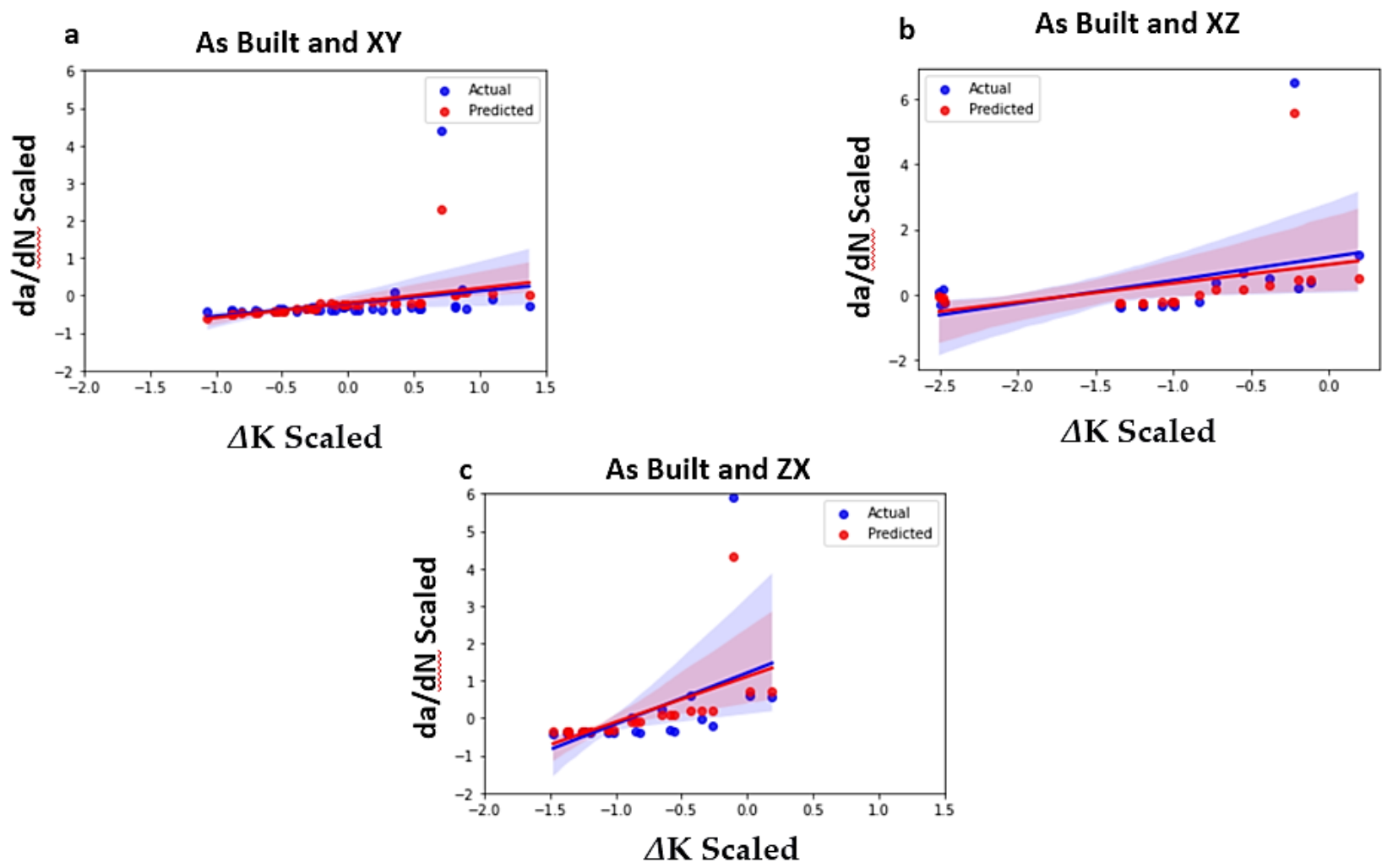

Figure 3. Actual and Prediction Plots for as Built Ti-64 alloy Samples. (a) XY Specimen Orientation; (b) XZ Specimen Orientation and (c) ZX Specimen Orientation.
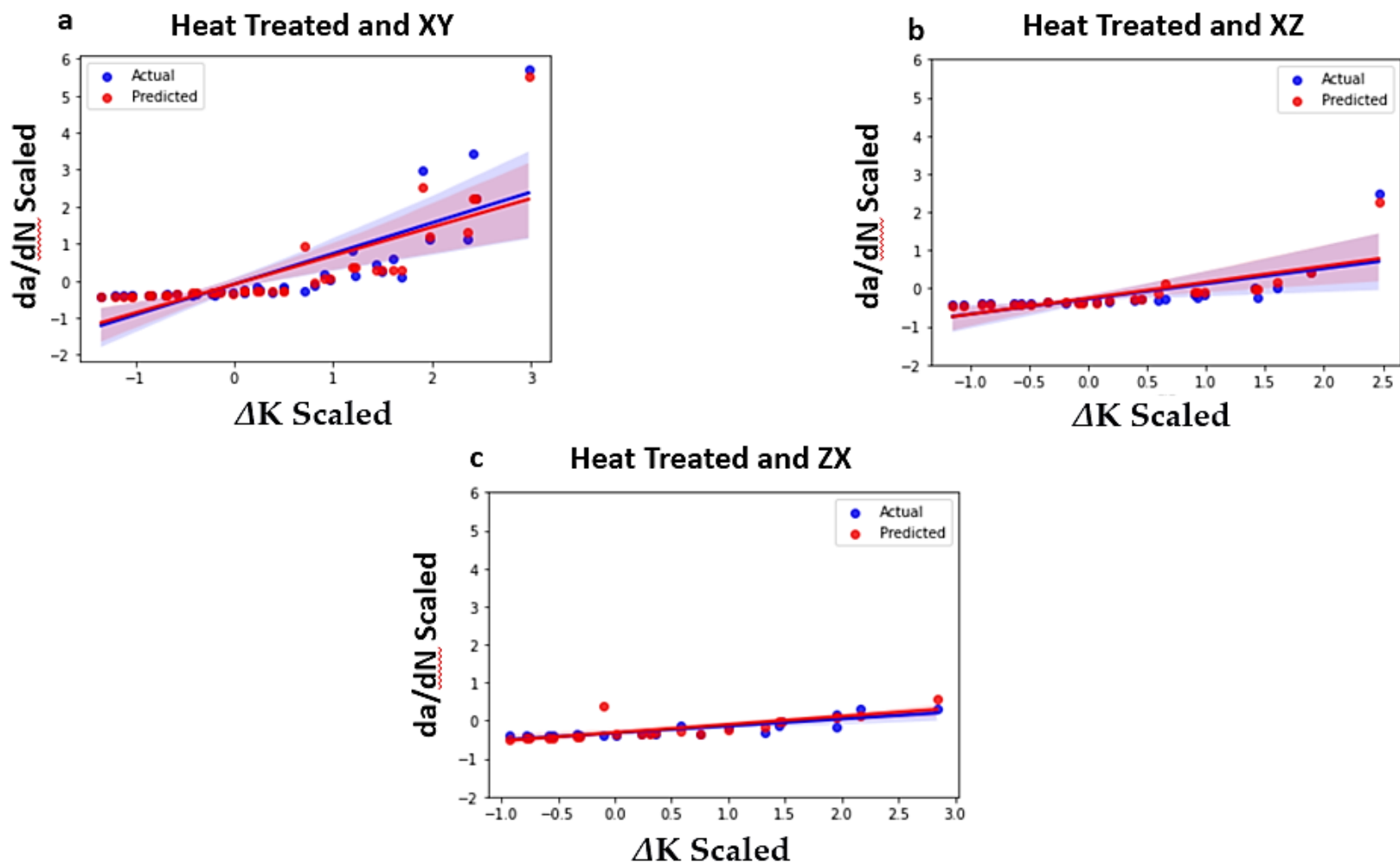

Figure 4. Actual and Prediction Plots for Heat treated Samples of Ti-64 alloy. (a) XY Specimen Orientation; (b) XZ Specimen Orientation and (c) ZX Specimen Orientation. 

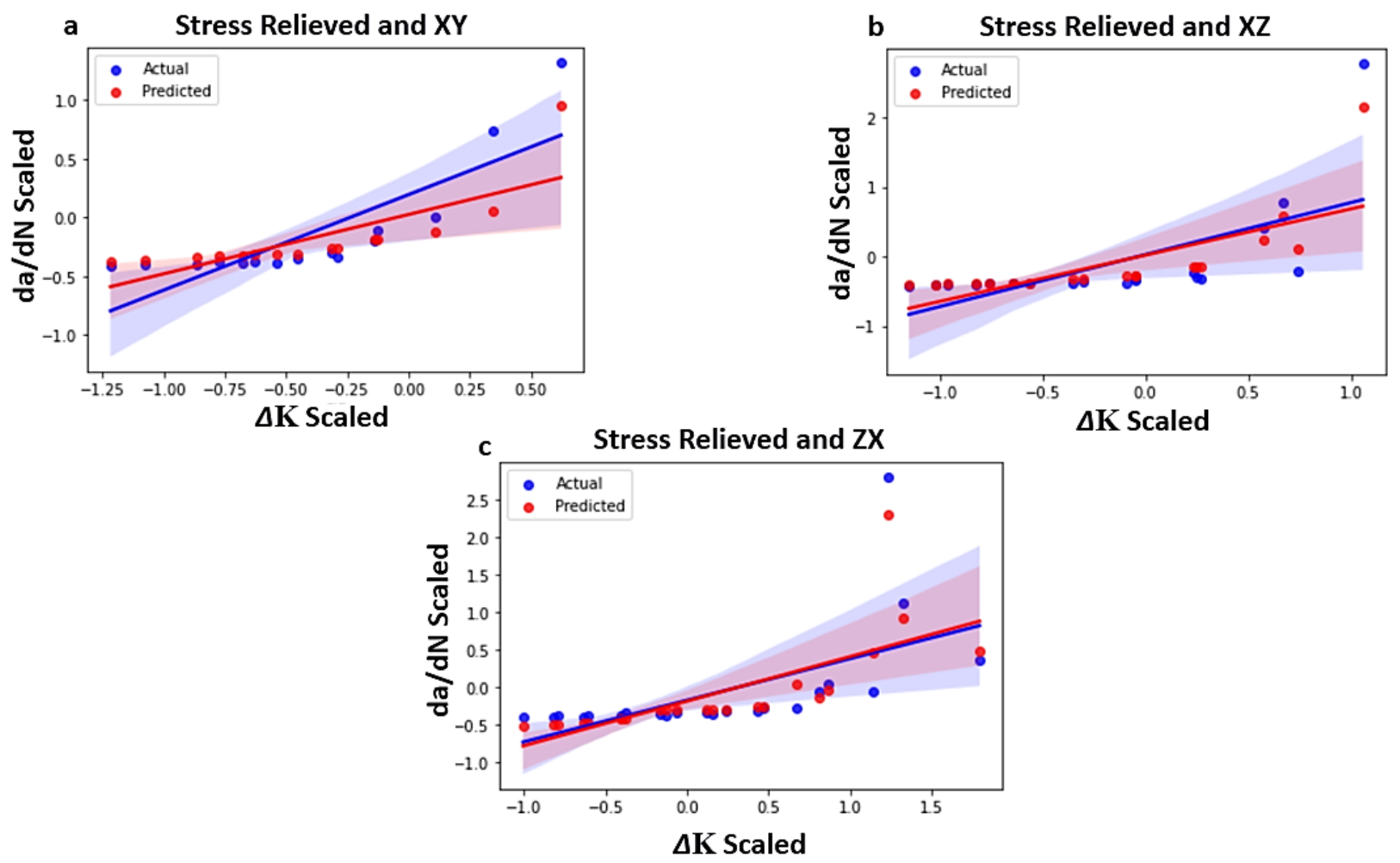

Figure 5. Actual and Prediction Plots for Stress Relieved Samples of Ti-64 alloy (a) XY Specimen Orientation; (b) XZ Specimen Orientation and (c) ZX Specimen Orientation.

From Figure 6, it is observed that the DT, RF, and XGB were much superior to KNN. The KNN is the rudimentary ML algorithm which predicts the values based on its nearest neighbors. Due to the non-linearity of the data set in the present work, decision treebased algorithms were able to predict the patterns. It could be such situations when traversing through non-linear data, wherein the data points in particular direction could be further away from other data points and therefore, this KNN algorithm tries to move in the direction where the data points are nearer. Therefore, the performance of KNN algorithm was not comparable to Decision tree-based algorithms for the data analyzed in the present work.

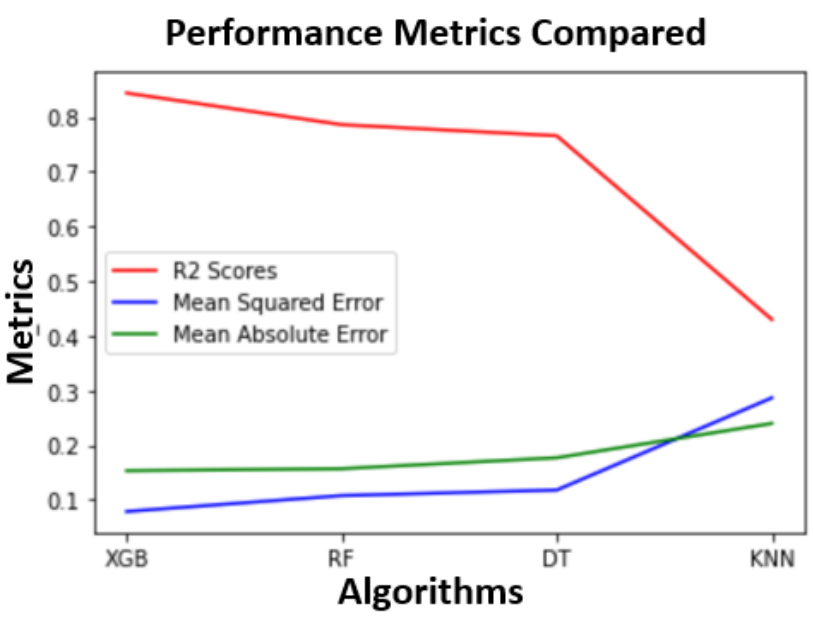

Figure 6. Comparison of Performance metrics of all the 4 different algorithms. 


\subsection{Feature Importance Analysis}

Feature Importance analysis is carried out to determine the most influencing independent variables on the target column. For the dataset chosen, the features considered are Post Processing Technique, built orientation and $\Delta K$. Figure 7 shows the result of the performed feature importance analysis. It is found that the most influencing feature is $\Delta \mathrm{K}$ as this is the testing condition having direct influence on FCGR through Paris law. However, the Post Processing parameter and Built Direction are the two conditions whose importance are analyzed by Feature Importance analysis for the chosen dataset. After the Hyper parameter tuning of XGB algorithm, the feature importance test was performed using the optimized hyper parameters. The importance was calculated based on each decision tree plotted and importance of each independent variable was taken from each decision tree and aggregated to get the final important features. For the prediction of FCGR of Ti6Al4V with 3 different post processing techniques and 3 different built orientations present in the dataset, the post-processing technique was found to be more important feature than the built orientation. As shown in the Figure 7, the feature with high F-score is more important when compared to other features.

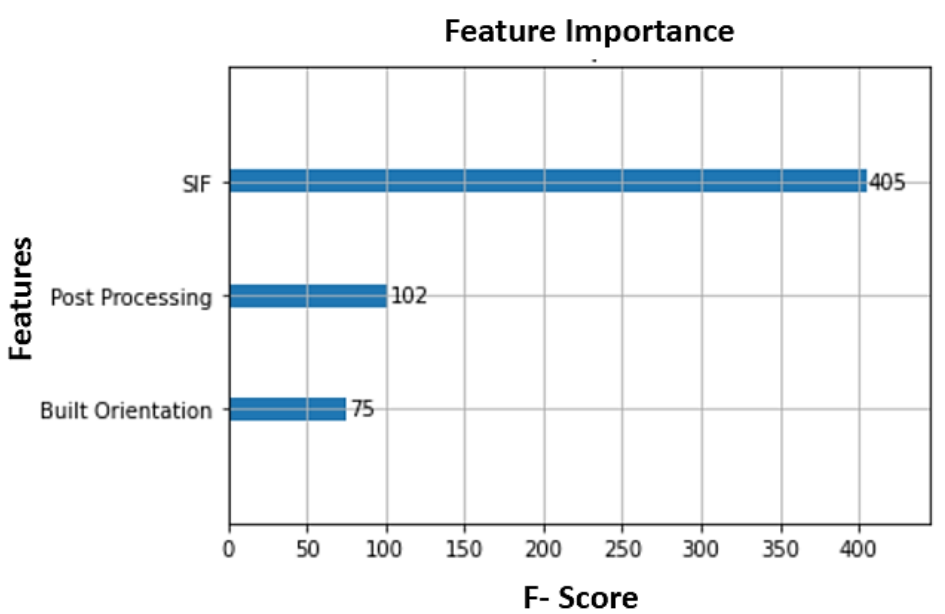

Figure 7. Feature Importance Ranking.

In predicting the FCGR of Ti6Al4V alloy manufactured using LPBF, Post Processing technique and Built Orientation are two independent parameters whose importance which is measured can be justified by the experimental analysis provided in the literature. For the as-built condition, $X Y$ oriented specimen demonstrates lowest FCGR, whereas the $\mathrm{ZX}$ and $\mathrm{XZ}$ oriented specimens demonstrated similar FCGR and higher compared to asbuilt $X Y$ oriented specimen. When the specimen is heat treated, the performance of $Z X$ and $\mathrm{XZ}$ is improved whereas $\mathrm{XY}$ oriented specimen performed slightly poorer. It is clear that in as-built condition the relation between the built directions is more noticeable. In other post processing conditions, the microstructural developments have occurred which is the function of heat treatment temperature and residual stresses are relieved which alter the FCGR. When the post processing is performed, it leads to the Hmogenization of microstructure and the Paris law curves for all the build directions approach closer to each other, where the significance of Build direction diminishes. Hence, Post Processing technique is more important feature when compared to Build Orientation.

It is shown that machine learning model is capable of predicting the experimental results accurately as well as to identify the influence of independent parameters appropriately.

\section{Microstructure-Property Correlation}

The fatigue crack growth behaviour of additively manufactured Ti alloys is highly microstructural dependent and therefore it is essential to discuss the microstructural constituents of the alloys influenced by post-processing (such as heat treatment cycles), AM built orientation, composition, etc., in this section. These microstructural features also 
influencing the static properties such as stress intensity factor (SIF), tensile, and hardness along with dynamic properties (fatigue). Hence, these influencing microstructure and associated property are accounted here to develop better understanding on ML assisted prediction of fatigue properties of the Ti6Al4V alloy.

The microstructural evolution of Ti alloys occurs based on thermodynamic kinetics principles. The Ti alloys are categorized mainly in terms of commercially pure (CP), $\alpha /$ near $\alpha, \alpha+\beta$, and $\beta$ types of alloys along with Ti-xAl type intermetallic compounds, which are utilized based on its microstructural characteristics and properties in different applications [36]. All the above constituent phases of the alloys are obtained primarily based on $\alpha / \beta$ transus temperatures, martensitic start $\left(\mathrm{M}_{\mathrm{s}}\right)$, martensitic finish $\left(\mathrm{M}_{\mathrm{f}}\right)$, and cooling rate parameters. The $\alpha$ alloys or super- $\alpha$ alloys contain major amount of $\alpha$ stabilizer with a lean amount of $\beta$ stabilizer to achieve good fatigue crack growth and creep resistance. Such alloys are widely reported and collected to utilize in FCGR prediction through ML modelling. Usually, such $\alpha$ alloys (hcp) exhibit suppressed ductility and toughness at cryogenic temperature but could be improved by reducing interstitial contents, known as Extra Low Interstitial (ELI) alloys. Along with creep properties, ELI grade $\alpha$ alloys possess excellent combination of ductility, toughness, and fatigue properties which are used extensively in engineering structural components. The Ti-5Al-2.5Sn is one of the ELI grades $\alpha$ Ti alloys exhibits high fatigue strength besides its good tensile properties. The Ti-6Al-2Sn-4Zr-2Mo-0.1Si, Ti-6242S(c)(e), IMI 685, IMI 829, and IMI 834 are the example of Super- $\alpha$ alloys which show good fatigue properties.

The addition of $\alpha$ and $\beta$ stabilizers would produce $\alpha+\beta$ dual phase Ti alloys which influences cyclic strength significantly and hence fatigue crack growth resistance too. Aluminum is the example of $\alpha$ stabilizer whereas vanadium or molybdenum are $\beta$ stabilizers. The sufficient presence of $\beta$ formers could assist to reach beyond $\beta$ transus temperature (e.g., $994{ }^{\circ} \mathrm{C}$ for Ti-64) while heating, where uniform single phase $\beta$ (bcc) is obtained. Cooling down from the $\beta$ transus, would help to retain $\beta$ phase or martensitic transformation. Such a solution treatment followed by aging would strengthen the $\alpha+\beta$ alloys through the formation of transformed- $\beta$ /martensite $\left(\alpha^{\prime}\right)$ and retained- $\beta$ /acicular- $\alpha$ which show higher high cycle fatigue strength and lower fatigue crack growth. The solution treatment is followed by quenching either through water, oil or suitable quenchant solution. The optimized process parameters in additive manufacturing could lead to $\alpha+\beta$ Ti alloys with the desired morphology for reducing the number of post-processing steps prior to its use. The $\alpha+\beta$ microstructure is dependent on alloy composition, solution treatment condition, cooling rate, and section size. Aging of $\alpha+\beta$ alloys are usually performed in between $480{ }^{\circ} \mathrm{C}$ to $650{ }^{\circ} \mathrm{C}$, which precipitate the $\alpha$ phase or fine $\alpha / \beta$ combinations in retained- $\beta$ phase. Also, the adequate solution treatment and aging of this alloy could lead to enhanced strength more that $30 \%$ which might be the reason for higher fatigue crack growth behaviour too [37-41]. The few $\alpha+\beta$ Ti alloys are Ti-6Al-4V, Ti-6Al-4V-ELI, Ti-6Al-6V-2Sn, UNS 56080, UNS 56740, Ti-6242, Ti-17, Ti-6Al-2Sn-2Zr-2Cr-2Mo, Ti-3Al-2.5V, Ti-8Al-1Mo-1V(e), etc.

\section{Conclusions}

Fatigue crack growth rate behaviour of Ti6Al4V alloy fabricated by LPBF with respect to 9 different specimen conditions was analyzed by using machine learning techniques. The following conclusions are made based on the present work.

1. The influence of postprocessing treatments and built orientation on and FCGR of Ti6Al4V fabricated using LPBF, analyzed through different ML algorithms, have shown that the former has influenced its fatigue life significantly compared to the latter.

2. It was observed that the XGB algorithm has led to best R2 score and least mean squared error in predicting the FCGR of Ti64 alloy.

3. In the feature importance analysis, apart from $\Delta \mathrm{K}$, the important parameters identified are Post Processing technique and Built Orientation for predicting the FCGR of Ti64 alloy. 
Since the optimization of processing and postprocessing parameters are still in developing stage in metal additive manufacturing, data-driven models can help in establishing the appropriate set of input variables. An effective property predictive model can improve the understanding of FCGR mechanisms which would help to design the materials with high performance.

Author Contributions: Conceptualization-N.K.; methodology-N.K. and R.J.; software-N.K.; validation-N.K.; formal analysis-N.K. and R.J.; investigation-N.K.; resources—R.J.; data curationN.K. and R.V.; writing — original draft preparation-N.K. and R.V.; writing—review and editing-R.J.; visualization-N.K. and R.J.; supervision-R.J.; project administration-R.J. All authors have read and agreed to the published version of the manuscript.

Funding: The authors acknowledge the funding support from the Ministry of Education through Institute of Eminence (IoE), IIT Madras.

Institutional Review Board Statement: Is not applicable for this study as it did not involve any human or animal subject.

Informed Consent Statement: Not applicable.

Data Availability Statement: https://github.com/nithink27/FCGR-Predictions-using-MachineLearning. Updated on 19 October 2021.

Conflicts of Interest: The authors declare no conflict of interest.

\section{References}

1. Jebieshia, T.R.; Kim, J.M.; Kang, J.W.; Son, S.W.; Kim, H.D. Microstructural and Very High Cycle Fatigue (VHCF) Behavior of Ti6Al4V—A Comparative Study. Materials 2020, 13, 1948. [CrossRef] [PubMed]

2. Bača, A.; Konečná, R.; Nicoletto, G.; Kunz, L. Influence of Build Direction on the Fatigue Behaviour of Ti6Al4V Alloy Produced by Direct Metal Laser Sintering. Mater. Today Proc. 2016, 3, 921-924. [CrossRef]

3. Kahlin, M.; Ansell, H.; Kerwin, A.; Smith, B.; Moverare, J. Variable amplitude loading of additively manufactured Ti6Al4V subjected to surface post processes. Int. J. Fatigue 2021, 142, 105945. [CrossRef]

4. Agius, D.J.; Kourousis, K.I.; Wallbrink, C. A Review of the As-Built SLM Ti-6Al-4V Mechanical Properties towards Achieving Fatigue Resistant Designs. Metals 2018, 8, 75. [CrossRef]

5. Günther, J.; Tröster, T.; Weidner, A.; Leuders, S. Fatigue behavior of Ti-6Al-4V additively manufactured by selective laser and electron beam melting-On the impact of the chemical composition, process- induced porosity and surface roughness. DVM-Rep. 2017, 2, 89-98.

6. Mertova, K.; Dzugan, J.; Roudnicka, M. Fatigue properties of SLM-produced Ti6Al4V with various post-processing processes. IOP Conf. Ser. Mater. Sci. Eng. 2018, 461, 012052. [CrossRef]

7. Dinh, T.; Han, S.; Yaghoubi, V.; Xiang, H.; Erdelyi, H.; Craeghs, T.; Segers, J.; Van Paepegem, W. Modeling detrimental effects of high surface roughness on the fatigue behavior of additively manufactured Ti-6Al-4V alloys. Int. J. Fatigue 2021, $144,106034$. [CrossRef]

8. Qian, M.; Xu, W.; Brandt, M.; Tang, H. Additive manufacturing and postprocessing of Ti-6Al-4V for superior mechanical properties. MRS Bull. 2016, 41, 775-784. [CrossRef]

9. Jamshidi, P.; Aristizabal, M.; Kong, W.; Villapun, V. Selective laser melting of Ti-6Al-4V: The impact of post-processing on the tensile, fatigue and biological properties for medical implant applications. Materials 2020, 13, 2813. [CrossRef] [PubMed]

10. Shen, B.; Li, H.; Liu, S.; Zou, J.; Shen, S.; Wang, Y.; Zhang, T.; Zhang, D.; Chen, Y.; Qi, H. Influence of laser post-processing on pore evolution of Ti-6Al-4V alloy by laser powder bed fusion. J. Alloys Compd. 2020, 818, 152845. [CrossRef]

11. Semenova, I.P.; Raab, G.I.; Golubovskiy, E.R.; Valiev, R.R. Service properties of ultrafine-grained Ti-6Al-4V alloy at elevated temperature. J. Mater. Sci. 2013, 48, 4806-4812. [CrossRef]

12. Hagiwara, M.; Kitashima, T.; Emura, S.; Iwasaki, S.; Shiwa, M. Very High-Cycle Fatigue and High-Cycle Fatigue of Minor Boron-Modified Ti-6Al-4V Alloy. Mater. Trans. 2019, 60, 2213-2222. [CrossRef]

13. Takeuchi, E.; Furuya, Y.; Nagashima, N.; Matsuoka, S. Effect of Stress Ratio on Giga-cycle Fatigue Properties for Ti-6Al-4V Alloy. Tetsu-to-Hagane 2010, 96, 36-41. [CrossRef]

14. Oguma, H.; Nakamura, T. The Effect of Stress Ratios on Very High Cycle Fatigue Properties of Ti-6Al-4V. Key Eng. Mater. 2004, 261-263, 1227-1232. [CrossRef]

15. Greitemeier, D.; Palm, F.; Syassen, F.; Melz, T. Fatigue performance of additive manufactured TiAl6V4 using electron and laser beam melting. Int. J. Fatigue 2017, 94, 211-217. [CrossRef]

16. Pessard, E.; Lavialle, M.; Laheurte, P.; Didier, P.; Brochu, M. High-cycle fatigue behavior of a laser powder bed fusion additive manufactured Ti-6Al-4V titanium: Effect of pores and tested volume size. Int. J. Fatigue 2021, 149, 106206. [CrossRef] 
17. Yakout, M.; Elbestawi, M.; Veldhuis, S.C. A study of the relationship between thermal expansion and residual stresses in selective laser melting of Ti-6Al-4V. J. Manuf. Process. 2020, 52, 181-192. [CrossRef]

18. Luo, Y.; Zhang, B.; Feng, X.; Song, Z.; Qi, X.; Li, C.; Chen, G.; Zhang, G. Pore-affected fatigue life scattering and prediction of additively manufactured Inconel 718: An investigation based on miniature specimen testing and machine learning approach. Mater. Sci. Eng. A 2020, 802, 140693. [CrossRef]

19. Macallister, N.; Vanmeensel, K.; Becker, T.H. Fatigue crack growth parameters of Laser Powder Bed Fusion produced Ti-6Al-4V. Int. J. Fatigue 2021, 145, 106100. [CrossRef]

20. González, J.; Armas, G.; Negrin, J.; Beltrán, A.; Trueba, P.; Gotor, F.; Peón, E.; Torres, Y. Influence of Successive Chemical and Thermochemical Treatments on Surface Features of Ti6Al4V Samples Manufactured by SLM. Metals 2021, 11, 313. [CrossRef]

21. Teixeira, Ó.; Silva, F.J.G.; Ferreira, P. Quality and Residual Stresses of the Ti-6Al-4V Parts Produced by Additive Manufacturing. Metals 2020, 10, 1006. [CrossRef]

22. Cain, V.; Thijs, L.; Van Humbeeck, J.; Van Hooreweder, B.; Knutsen, R. Crack propagation and fracture toughness of Ti6Al4V alloy produced by selective laser melting. Addit. Manuf. 2015, 5, 68-76. [CrossRef]

23. Thijs, L.; Verhaeghe, F.; Craeghs, T.; Van Humbeeck, J.; Kruth, J.-P. A study of the microstructural evolution during selective laser melting of Ti-6Al-4V. Acta Mater. 2010, 58, 3303-3312. [CrossRef]

24. Raja, A.; Chukka, S.T.; Jayaganthan, R. Prediction of Fatigue Crack Growth Behaviour in Ultrafine Grained Al 2014 Alloy Using Machine Learning. Metals 2020, 10, 1349. [CrossRef]

25. Nguyen-Le, D.; Do, D.T.T.; Lee, J.; Rabczuk, T.; Nguyen-Xuan, H. Forecasting Damage Mechanics by Deep Learning. Comput. Mater. Contin. 2019, 61, 951-977. [CrossRef]

26. Wang, B.; Zhao, W.; Du, Y.; Zhang, G.; Yang, Y. Prediction of fatigue stress concentration factor using extreme learning machine Comput. Mater. Sci. 2016, 125, 136-145. [CrossRef]

27. Zhan, Z.; Li, H. A novel approach based on the elastoplastic fatigue damage and machine learning models for life prediction of aerospace alloy parts fabricated by additive manufacturing. Int. J. Fatigue 2021, 145, 106089. [CrossRef]

28. Zhan, Z.; Li, H. Machine learning based fatigue life prediction with effects of additive manufacturing process parameters for printed SS 316L. Int. J. Fatigue 2021, 142, 105941. [CrossRef]

29. Moon, S.; Ma, R.; Attardo, R.; Tomonto, C.; Nordin, M.; Wheelock, P.; Glavicic, M.; Layman, M.; Billo, R.; Luo, T. Impact of surface and pore characteristics on fatigue life of laser powder bed fusion Ti-6Al-4V alloy described by neural network models. Sci. Rep. 2021, 11, 1-17. [CrossRef]

30. Du, L.; Qian, G.; Zheng, L.; Hong, Y. Influence of processing parameters of selective laser melting on high-cycle and very-highcycle fatigue behaviour of Ti-6Al-4V. Fatigue Fract. Eng. Mater. Struct. 2021, 44, 240-256. [CrossRef]

31. Pan, X.; Li, X.; Zhou, L.; Feng, X.; Luo, S.; He, W.; Semenova, I.P.; Raab, G.I.; Golubovskiy, E.R.; Valiev, R.Z.R.; et al. Surface roughness effects on the fatigue strength of additively manufactured Ti-6Al-4V. Int. J. Fatigue 2017, 116, 543-552. [CrossRef]

32. Wycisk, E.; Emmelmann, C.; Siddique, S.; Walther, F. High Cycle Fatigue (HCF) Performance of Ti-6Al-4V Alloy Processed by Selective Laser Melting. Adv. Mater. Res. 2013, 816-817, 134-139. [CrossRef]

33. Xie, Y.; Gong, M.; Zhou, Q.; Li, Q.; Wang, F.; Zeng, X.; Gao, M. Effect of microstructure on fatigue crack growth of wire arc additive manufactured Ti-6Al-4V. Mater. Sci. Eng. A 2021, 826, 141942. [CrossRef]

34. Kamble, R.G.; Raykar, N.; Jadhav, D. Machine learning approach to predict fatigue crack growth. Mater. Today Proc. 2021, 38 , 2506-2511. [CrossRef]

35. Mythreyi, O.; Srinivaas, M.; Kumar, T.A.; Jayaganthan, R. Machine-Learning-Based Prediction of Corrosion Behavior in Additively Manufactured Inconel 718. Data 2021, 6, 80. [CrossRef]

36. Cui, C.; Hu, B.; Zhao, L.; Liu, S. Titanium alloy production technology, market prospects and industry development. Mater. Des. 2011, 32, 1684-1691. [CrossRef]

37. Galarraga, H.; Warren, R.; Lados, D.; Dehoff, R.R.; Kirka, M.M.; Nandwana, P. Effects of heat treatments on microstructure and properties of Ti-6Al-4V ELI alloy fabricated by electron beam melting (EBM). Mater. Sci. Eng. A 2017, 685, 417-428. [CrossRef]

38. Ahmed, T.; Rack, H. Phase transformations during cooling in $\alpha+\beta$ titanium alloys. Mater. Sci. Eng. A 1998, $243,206-211$. [CrossRef]

39. Banerjee, D.; Williams, J.C. Perspectives on Titanium Science and Technology. Acta Mater. 2013, 61, 844-879. [CrossRef]

40. Liu, S.; Shin, Y.C. Additive manufacturing of Ti6Al4V alloy: A review. Mater. Des. 2019, 164, 107552. [CrossRef]

41. Leuders, S.; Lieneke, T.; Lammers, S.; Tröster, T.; Niendorf, T. On the fatigue properties of metals manufactured by selective laser melting-The role of ductility. J. Mater. Res. 2014, 29, 1911-1919. [CrossRef] 